\title{
Coding Gain in Paraunitary Analysis/Synthesis Systems
}

\author{
Anand K. Soman, Student Member, IEEE, and P. P. Vaidyanathan, Fellow, IEEE
}

\begin{abstract}
Subband coders have been used in the past to decompose a signal into subbands. The signals in each subband are quantized before transmission. The problem of optimal bit allocation involves allocating bits to the individual quantizers from a fixed budget so as to minimize the overall reconstruction error variance. The problem has been addressed in the past for two cases, namely orthogonal transform coding, and ideal brick-wall filtering. Both of these are special cases of the socalled "paraunitary" filter banks. The results which were proved for these special cases have been used without proof for other nonparaunitary subband coding schemes. We present here a formal proof that these bit-allocation results hold for the entire class of paraunitary subband coders. Next, we address the problem of finding an optimal paraunitary subband coder, so as to maximize the coding gain of the system.

We then analyze the bit-allocation problem for the case of paraunitary tree-structured filter banks, such as those used for generating orthonormal wavelets. The even more general case of nonuniform filter banks is next considered. In all cases we show that under optimal bit allocation, the variance of the errors introduced by each of the quantizers have to be equal. Expressions for coding gains for these systems have also been derived.
\end{abstract}

\section{INTRODUCTION}

$\mathrm{T}$ RANSFORM coding and subband coding are wellknown techniques for efficiently encoding data [1][5]. They are used in data compression of speech, image, and other random signals. Consider the subband coding scheme shown in Fig. 1. In this scheme, the input signal $x(n)$ is split into $M$ subbands in the frequency domain by a bank of filters called the analysis filters. The outputs of these filters are band limited, and hence we can subsample them. This is indicated by boxes with $\downarrow M$. The signals in each of the subbands are then independently quantized and transmitted. At the receiving end, the sampling rates in each of the subbands are increased once again to their original value by the interpolaters (indicated by $\uparrow M$ ). They are then passed through the synthesis filters. The outputs of the synthesis filters are combined to give the reconstructed signal $y(n)$.

Another way of representing the same scheme is shown in Fig. 2. In this figure, $\boldsymbol{E}(z)$ and $\boldsymbol{R}(z)$ are the polyphase

Manuscript received May 20, 1991; revised July 21, 1992. The associate editor coordinating the review of this paper and approving it for publication was Prof. Faye Boudreaux-Bartels. This work was supported in part by NSF Grant MIP 8919196. Tektronix Inc., and Rockwell International.

The authors are with the Department of Electrical Engineering, California Institute of Technology, Pasadena, CA 91125.

IEEE Log Number 9207532

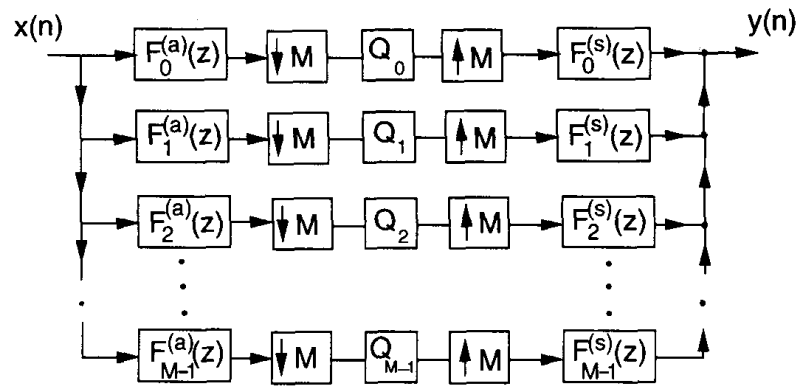

Fig. 1. A typical subband coding scheme.

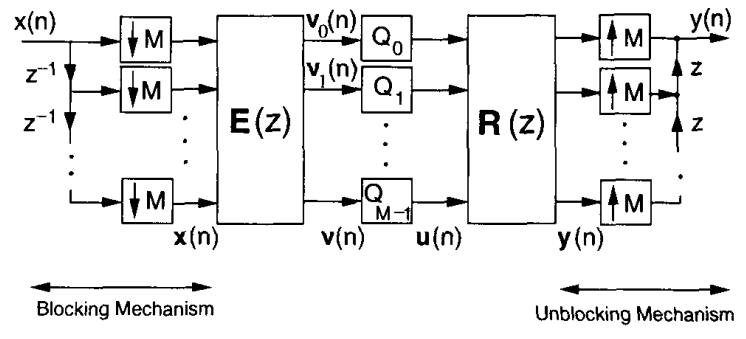

Fig. 2. Subband coding scheme showing polyphase matrices.

matrices [6] corresponding to the analysis and synthesis filters, respectively. The sequence $x(n)$ is divided into nonoverlapping blocks of data by grouping together $M$ successive samples. These samples form the components of the vector $\boldsymbol{x}(n)$ which is termed the $M$-fold blocked version of $x(n)$. Formally, we write

$$
\boldsymbol{x}^{T}(n)=[x(n M) x(n M-1) \cdots x(n M-M+1)] .
$$

Each of these blocks or vectors is encoded by the linear transformation $\boldsymbol{E}(z)$. The $M$ outputs, i.e., the components of the vector $v(n)$ are the subband signals which are quantized and transmitted. At the receiver, the received vector $\boldsymbol{u}(n)$ is passed through the transformation $\boldsymbol{R}(z)$. The output $y(n)$ is "unblocked" to give the reconstructed sequence $y(n)$. A special case of this scheme is the transform coding scheme [1], [2] which we shall now review.

\section{A. Transform Coding}

In a traditional transform coding scheme, the polyphase matrices $\boldsymbol{E}(z)$ and $\boldsymbol{R}(z)$ mentioned earlier are chosen to be 
constant matrices. The matrix at the transmitting end is often chosen to be a orthogonal matrix ( $A$ say), so that if the matrix $\boldsymbol{A}^{T}$ is used at the receiver, the system becomes a perfect reconstruction system (i.e., $y(n)=x(n)$ ) in the absence of quantizers. In the presence of quantizers, a natural objective in such a coding system is to minimize the reconstruction error between the input and the output. The variance of the reconstruction error is chosen as a suitable criterion for minimization [2]. Define the error vector to be the difference between the input vector and the output vector, i.e.,

$$
r(n)=\boldsymbol{y}(n)-\boldsymbol{x}(n) .
$$

Assuming this error to be a zero-mean, wide-sense stationary (abbreviated WSS) vector process, the reconstruction error variance is

$$
\sigma_{r}^{2}=\left(\frac{1}{M}\right) E\left[r^{T}(n) r(n)\right] .
$$

In a conventional pulse code modulation (PCM) scheme, the input samples are independently quantized and transmitted over the channel. This is equivalent to making the transform matrix in Fig. 2 an identity matrix. The coding gain [2] of the transform coding system is defined as the ratio of the error variance in a PCM system to the error variance in the transform coding system, i.e.,

$$
G_{T C}=\frac{\sigma_{r \cdot \mathrm{PCM}}^{2}}{\sigma_{r \cdot T C}^{2}} .
$$

Now let us turn to the individual quantizers. Let $R_{i}$ be the number of bits allocated to the quantizer $Q_{i}$ and let $\sigma_{t^{\prime} j}^{2}$ be the variance of the input to that quantizer. The range of values that the input to the quantizer can take is divided into $2^{R_{i}}$ intervals. The weight of the most significant bit is taken to be proportional to $\sigma_{l^{\prime} i}$, so that the probability of overflow is the same for each $i$. The variance of the error introduced by the $i$ th quantizer is then given as [2]

$$
\sigma_{i}^{2}=\epsilon^{2} 2^{-2 R_{i}} \sigma_{i i}^{2}
$$

where $\epsilon$ is a constant. Now, suppose the total number of bits available for quantizing all the $M$ subband signals is fixed, i.e.,

$$
R=\left(R_{0}+R_{1}+R_{2}+\cdots+R_{M-1}\right) / M=\text { constant. }
$$

The design issues in the transform coding scheme then are:

1) How does one allocate bits to the individual quantizers under the constraint imposed by (1.6), and

2) How does one choose the orthogonal transformation $\boldsymbol{A}$ so as to maximize the coding gain of the system?

In the case of the transform coding schemes it has been shown [1], [2] that the optimal allocation of bits is that which makes all the individual quantizer error variances equal. Under optimal bit allocation, it has also been shown
[2], that the coding gain of the system becomes

$$
G_{T C}=\frac{(1 / M)\left(\sum_{k=0}^{M-1} \sigma_{v_{k}}^{2}\right)}{\left(\prod_{k=0}^{M-1} \sigma_{v_{k}}^{2}\right)^{1 / M}}
$$

which is the ratio of the arithmetic mean to the geometric mean of the $\sigma_{i k}^{2}$. This is maximized if the transform matrix $\boldsymbol{A}$ is the Karhunen-Loeve transform (KLT) [2].

\section{B. Paraunitary Filter Banks}

A linear transformation $\boldsymbol{E}(z)$ is said to be paraunitary if it satisfies [6]

$$
\tilde{E}(z) \boldsymbol{E}(z)=\boldsymbol{I}
$$

where $\tilde{E}(z)$ is obtained from $\boldsymbol{E}(z)$ by conjugating, transposing, and replacing $z$ by $z^{-1}$. The paraunitary property is essentially an extension of the unitary property to linear, time-invariant systems with memory. Paraunitary transformations are important in subband coding because the subband coding system shown in Fig. 2 can be made to have perfect reconstruction property (in the absence of quantizers) by choosing the matrix $\boldsymbol{E}(z)$ to be paraunitary, and choosing $\boldsymbol{R}(z)$ to be $\tilde{E}(z)$ [7]. The analysis and synthesis filters are then related as $F_{k}^{(s)}(z)=$ $\tilde{F}_{k}^{(a)}(z)$. Second, it has been shown [6] that paraunitary transformations can be realized using lattice/cascade structures. In the case of the paraunitary subband coding system we define the coding gain in a likewise manner, i.e.,

$$
G_{\mathrm{PU}}=\frac{\sigma_{r . \mathrm{PCM}}^{2}}{\sigma_{r . \mathrm{PU}}^{2}} .
$$

One can ask questions similar to those asked before, namely,

1) How does one allocate bits to the quantizers, and

2) How does one choose the paraunitary transformation $\boldsymbol{E}(z)$ so as to maximize the coding gain?

The transform coding scheme discussed previously is one special case of paraunitary subband coders. Now consider ideal subband coders (using brickwall filters as shown in [4, fig.7.46]. This can also be shown (by invoking $[7$, eq. (36)] to be a special case of paraunitary subband coders. In this case too, under optimal bit allocation, (1.7) holds [2]. Equation (1.7) has been used without proof in the context of lapped orthogonal transform (LOT) in [8] also, which are a special case of paraunitary systems.

The problem of optimal bit allocation itself has been mentioned in [3], [9]-[11] for nonparaunitary subband coders. In this paper, we derive conditions for bit-allocation optimality of general paraunitary subband coders, and formally prove that a result similar to (1.7) holds for this entire class. We also address the problem of finding the optimal paraunitary transformation so as to maximize the coding gain. Next we consider the bit-allocation prob- 
lem in the context of paraunitary tree-structured filter banks such as those used for generating wavelets [12], [13]. Our final extension of this analysis is to the case of general non-uniform filter banks.

Notations and Definitions: The notations used in this paper are as follows: Boldfaced quantities denote matrices and vectors, as in $\boldsymbol{E}$ or $\boldsymbol{x} \cdot \boldsymbol{A}^{T}$ denotes the transpose of the matrix $A . \operatorname{tr}(\boldsymbol{A})$ denotes the trace of the matrix $A$, i.e., it is the sum of the diagonal terms of the matrix. $\operatorname{det}(\boldsymbol{A})$ denotes the determinant of the matrix $\boldsymbol{A}$. The tilde notation, as in $\tilde{E}(z)$, stands for conjugation of coefficients followed by transposition, followed by replacing $z$ by $z^{-1}$. As defined earlier, a matrix $\boldsymbol{E}(z)$ is said to be paraunitary if it satisfies (1.8). Note that if $\boldsymbol{E}(z)$ is a square paraunitary matrix, it also satisfies

$$
\boldsymbol{E}(z) \tilde{E}(z)=\boldsymbol{I} .
$$

We shall deal with FIR matrices with real coefficients. The FIR nature is required if we constrain both the analysis and synthesis filters to be stable [6]. Let $N$ be the order of $\boldsymbol{E}(z)$

$$
\boldsymbol{E}(z)=\boldsymbol{e}(0)+\boldsymbol{e}(1) z^{-1}+\boldsymbol{e}(2) \mathrm{z}^{-2}+\cdots+\boldsymbol{e}(N) z^{-N} .
$$

Therefore,

$$
\tilde{E}(z)=\boldsymbol{e}^{T}(0)+\boldsymbol{e}^{T}(1) z+\boldsymbol{e}^{T}(2) z^{2}+\cdots+\boldsymbol{e}^{T}(N) z^{N} .
$$

Expressed in time-domain the square paraunitary relations (1.8) and (1.10) imply

$$
\begin{aligned}
& \sum_{m} \boldsymbol{e}^{T}(m-l) \boldsymbol{e}(m)=\delta(l) \boldsymbol{I} \\
& \sum_{m} \boldsymbol{e}(m-l) \boldsymbol{e}^{T}(m)=\delta(l) \boldsymbol{I} .
\end{aligned}
$$

All signals considered are real. A vector random process $x(n)$ is said to be WSS if $E[x(n)]=\boldsymbol{m}_{x}$, independent of $n$, and

$$
E\left[x(n) x^{T}(n-k)\right]=\boldsymbol{R}(k) \quad \text { for all } n, k .
$$

A random process $x(n)$ is cyclo-wide-sense stationary with a period $M$, abbreviated as (CWSS) $)_{M}$, if its $M$-fold blocked version $\boldsymbol{x}(n)$ as defined in (1.1) is a WSS vector process. Conversely, if $x(n)$ is a WSS vector process, then the sequence $x(n)$ obtained by unblocking it is (CWSS $)_{M}$. A vector process $p(n)$ of size $M L$ is said to be a $M$-fold blocked version of another vector process $s(n)$ of size $L$ if they are related as

$$
p^{T}(n)=\left[s^{T}(n M) s^{T}(n M-1) \cdots s^{T}(n M-M+1)\right] .
$$

We say a vector process $s(n)$ is (CWSS) $)_{M}$ if its $M$-fold blocked version $p(n)$ as defined above is a WSS vector process. Let $\boldsymbol{R}(z)$ be a multi-input multi-output system. The matrix $\boldsymbol{B}(z)$ is called the $M$-fold blocked version of the matrix $R(z)$ if

$\boldsymbol{B}(z)$

$$
=\left[\begin{array}{llclc}
\boldsymbol{R}_{0}(z) & \boldsymbol{R}_{1}(z) & \boldsymbol{R}_{2}(z) & \cdots & \boldsymbol{R}_{M-1}(z) \\
z^{-1} \boldsymbol{R}_{M-1}(z) & \boldsymbol{R}_{0}(z) & \boldsymbol{R}_{1}(z) & \cdots & \boldsymbol{R}_{M-2}(z) \\
z^{-1} \boldsymbol{R}_{M-2}(z) & z^{-1} \boldsymbol{R}_{M-1}(z) & \boldsymbol{R}_{0}(z) & \cdots & \boldsymbol{R}_{M-3}(z) \\
\vdots & \vdots & \vdots & \ddots & \vdots \\
z^{-1} \boldsymbol{R}_{1}(z) & \cdots & \cdots & \cdots & \boldsymbol{R}_{0}(z)
\end{array}\right]
$$

where $\boldsymbol{R}_{i}(z)$ are the polyphase components [6] of the original matrix $\boldsymbol{R}(z)$, given by

$$
\begin{aligned}
\boldsymbol{R}(z)= & \boldsymbol{R}_{0}\left(z^{M}\right)+z^{-1} \boldsymbol{R}_{1}\left(z^{M}\right)+z^{-2} \boldsymbol{R}_{2}\left(z^{M}\right) \\
& +\cdots+z^{-(M-1)} \boldsymbol{R}_{M-1}\left(z^{M}\right) .
\end{aligned}
$$

The reason for calling $\boldsymbol{B}(z)$ the blocked version of $\boldsymbol{R}(z)$ is as follows. Let $s(n)$ be an input to the system $\boldsymbol{R}(z)$, and let $y(n)$ be the corresponding output. Let $p(n)$ be the $M$-fold blocked version of $s(n)$ as in (1.16), and let $y_{B}(n)$ be the $M$-fold blocked version of $y(n)$. Then it can be verified that the input $\boldsymbol{p}(n)$ to the system $\boldsymbol{B}(z)$ produces the output $\boldsymbol{y}_{B}(n)$. The proof of this when $\boldsymbol{R}(z)$ is a scalar can be found in [14], and the case where $R(z)$ is a matrix is a straightforward generalization of this proof.

In the figures, the acronym MIMO stands for multiinput, multi-output. LTI stands for linear time invariant. Boxes with $\downarrow \mathrm{M}$ and $\uparrow L$ stand for decimation by a factor $M$ and interpolation by a factor $L$, respectively, as defined in [6].

\section{Paraunitary Subband Coders}

The two design issues can be considered separately. First, we present a strategy for optimal bit allocation so as to minimize the reconstruction error variance. This bit allocation will hold irrespective of the paraunitary transformation used. We will then deal with the problem of finding an optimal FIR paraunitary transformation $E(z)$.

\section{A. Bit Allocation Result}

First we prove the following lemma:

Lemma 1: Consider a paraunitary multi-input multioutput system $\boldsymbol{E}(z)$. Let $\boldsymbol{x}(n)$, the input to this system, be a zero-mean vector WSS process, and let $y(n)$ be the output vector. Then,

$$
E\left[\boldsymbol{x}^{T}(n) \boldsymbol{x}(n)\right]=E\left[\boldsymbol{y}^{T}(n) \boldsymbol{y}(n)\right] .
$$

Proof: Let $S_{x x}\left(e^{j \omega}\right)$ and $S_{y y}\left(e^{j \omega}\right)$ be the power spectra of the vector sequences $\boldsymbol{x}(n)$ and $\boldsymbol{y}(n)$. Then,

$$
\boldsymbol{S}_{y \boldsymbol{y}}\left(e^{j \omega}\right)=\boldsymbol{E}\left(e^{j \omega}\right) \boldsymbol{S}_{x x}\left(e^{j \omega}\right) \boldsymbol{E}^{\dagger}\left(e^{j \omega}\right) .
$$

Since

$$
\operatorname{tr}\left[\boldsymbol{E}\left(e^{j \omega}\right) \boldsymbol{S}_{x x}\left(e^{j \omega}\right) \boldsymbol{E}^{\dagger}\left(e^{j \omega}\right)\right]=\operatorname{tr}\left[\boldsymbol{E}^{\dagger}\left(e^{j \omega}\right) \boldsymbol{E}\left(e^{j \omega}\right) \boldsymbol{S}_{x x}\left(e^{j \omega}\right)\right]
$$


we have

$$
\operatorname{tr}\left[S_{y y}\left(e^{j \omega}\right)\right]=\operatorname{tr}\left[S_{x x}\left(e^{j \omega}\right)\right] .
$$

Integrating the diagonal terms on both sides of the above equation gives us the required result.

Now consider the system shown in Fig. 2. $x(n)$ and $y(n)$, both vectors of size $M$, are the blocked versions of $x(n)$ and $y(n)$, respectively. Let the quantizers be modeled as zero-mean, WSS noise sources, uncorrelated with the input. Note that we do not assume the noise sources to be white or mutually uncorrelated. Let the total number of bits allocated to all the quantizers be fixed (1.6).

Theorem 1: Let $\boldsymbol{E}(z)$ be a FIR paraunitary matrix and let $\boldsymbol{R}(z)=\tilde{\boldsymbol{E}}(z)$. Then the reconstruction error variance of the system in Fig. 2 is minimized when the variances $\sigma_{i}^{2}$ of the errors introduced by each of the quantizers are equal, i.e., $\sigma_{i}^{2}=\sigma^{2}$ for all $i$.

Proof: Let $\boldsymbol{q}(n)=\boldsymbol{u}(n)-\boldsymbol{v}(n)$ and $\boldsymbol{r}(n)=\boldsymbol{y}(n)-$ $\boldsymbol{x}(n)$. Here, $\boldsymbol{q}(n)$ is the vector whose individual components are the quantizer errors, and $r(n)$ is the reconstruction error vector. So $\boldsymbol{r}(n)$ is the output of $\tilde{\boldsymbol{E}}(z)$ in response to $q(n)$, or, equivalently, $q(n)$ is the output of the system $\boldsymbol{E}(z)$ in response to the input $r(n)$. Hence using (1.11) we have

$$
\boldsymbol{q}(n)=\sum_{m=0}^{N} \boldsymbol{e}(m) r(n-m) .
$$

Therefore, applying Lemma 1,

$$
E\left[q^{T}(n) q(n)\right]=E\left[r^{T}(n) r(n)\right] .
$$

From the definition of $q(n)$, we have

$$
E\left[\boldsymbol{q}^{T}(n) \boldsymbol{q}(n)\right]=\sum_{i=0}^{M-1} \sigma_{i}^{2}
$$

so that $E\left[r^{T}(n) r(n)\right]=\sum_{i=0}^{M-1} \sigma_{i}^{2}$. Our problem is therefore to minimize $\Sigma_{i=0}^{M-1} \sigma_{i}^{2}$. Since the $\sigma_{i,}^{2}$ depend only on the input statistics and the paraunitary transformation $\boldsymbol{E}(z)$, we can show (using (1.5)) that (1.6) is equivalent to the condition $\left(\Pi_{i=0}^{M-1} \sigma_{i}^{2}\right)^{1 / M}=$ constant. We know [15] that the arithmetic mean of a set of nonnegative numbers is always greater than or equal to their geometric mean, i.e.,

$$
\sum_{i=0}^{M-1} \sigma_{i}^{2} \geq M\left(\prod_{i=0}^{M-1} \sigma_{i}^{2}\right)^{1 / M}=M c^{1 / M}
$$

with equality if and only if the $\sigma_{i}^{2}$ are the same for all $i$. Thus the above $\Sigma \sigma_{i}^{2}$ in $(2.6)$ is minimized if and only if all the $\sigma_{i}^{2}$ are equal, i.e.,

$$
\sigma_{1}^{2}=\sigma_{2}^{2}=\sigma_{3}^{2}=\cdots=\sigma_{M-1}^{2}
$$

We can therefore show that the following is the optimal bit allocation:

$$
R_{i}=R+(1 / 2) \log _{2}\left[\frac{\sigma_{v_{i}}^{2}}{\left(\prod_{i=0}^{M-1} \sigma_{v_{i}}^{2}\right)^{1 / M}}\right]
$$

In [2], [3], it was shown that the above equation holds for two special cases of paraunitary transformations. One must remember that it assumes high bit rates.

\section{B. Optimal Paraunitary Transforms}

This problem has been mentioned in literature for a few special types of paraunitary transformations. In [8], the author has dealt with the problem in context of the LOT, which are degree one paraunitary transformations with a particular form. A more recent work [16] deals with the extended lapped transform (ELT) which are paraunitary transformations of higher degrees, but again constrained to take a special form. By optimizing the filter responses, the author demonstrates coding gains approaching those of filter banks with ideal (brick-wall) filters. However, optimizing the filter responses is not necessarily the appropriate strategy, because ideal filters need not necessarily maximize the coding gain. To see this consider, by way of an example, a power spectral density which is as shown in Fig. 3. A two channel filter bank with brickwall filters gives a coding gain of unity, whereas a filter bank with filters $F_{1}^{(a)}(z)=1+z^{-1}$ and $F_{2}^{(a)}(z)=1-z^{-1}$ gives a coding gain of 1.0238 .

In [8], the problem of finding the optimal basis functions for the LOT so as to mazimize the coding gain has been formulated as a constrained optimization problem, to be solved by the method of Lagrange multipliers. The optimum basis functions are found in a sequential manner. However, it is not clear that such a sequential optimization would yield a global minimum. Another open problem therein is whether or not the optimal basis functions are the eigenvectors of the so-called extended autocorrelation matrix, as they are in the case of the KLT. The extended autocorrelation matrix is the autocorrelation matrix of size $M L$, corresponding to the input sequence, where $L-1$ is the order of the paraunitary transformation. We have the following proposition:

Proposition 2.1: The optimal basis functions are not necessarily the eigenvectors of the extended autocorrelation matrix.

Proof: Consider a 2-channel paraunitary subband coding system. We know that the extended autocorrelation matrix is positive definite and Toeplitz. It is easy to construct a positive-definite Toeplitz matrix with distinct eigenvalues. Hence it is a valid autocorrelation matrix of some process. Its eigenvectors are either symmetric or antisymmetric, i.e., the resulting filters have to be linear phase. However, we know that [6] a two-channel linear phase paraunitary subband coding system can only have trivial filters.

We suggest the following scheme for directly finding an optimal paraunitary transform so as to maximize the coding gain.

We have, $\sigma_{r, \mathrm{PU}}^{2}=(1 / M) \sum_{i=0}^{M-1} \sigma_{i}^{2}$ which under optimal bit allocation becomes $\left(\Pi_{i=0}^{M-1} \sigma_{i}^{2}\right)^{1 / M}$. Therefore, using (1.5) we get

$$
\sigma_{r, \mathrm{PU}}^{2}=\epsilon^{2} 2^{-2 R}\left(\prod_{i=0}^{M-1} \sigma_{n^{i_{i}}}^{2}\right)^{1 / M} .
$$




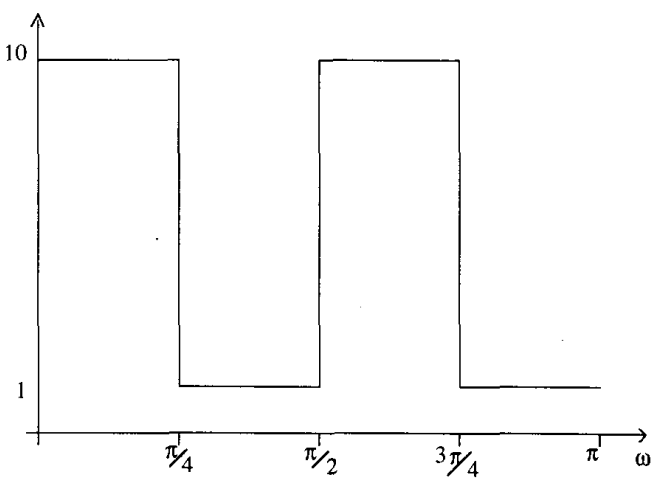

Fig. 3. Example of power spectrum for which brick-wall filters give zero (dB) coding gain.

Now,

$$
\sigma_{r, \mathrm{PCM}}^{2}=\epsilon^{2}(1 / M) 2^{-2 R} \sum_{i=0}^{M-1} \sigma_{r_{i}}^{2}
$$

and hence the coding gain of the paraunitary system becomes

$$
G_{\mathrm{PU}}=\frac{(1 / M)\left(\sum_{i=0}^{M-1} \sigma_{v^{2}}^{2}\right)}{\left(\prod_{i=0}^{M-1} \sigma_{v^{\prime}}^{2}\right)^{1 / M}}
$$

where $\sigma_{i,}^{2}$ is the variance of the input to the $i$ th quantizer. Note that this is true only for paraunitary transform matrices, and not for arbitrary subband coders. Both the brick-wall subband coder and the orthogonal transform coder satisfy this, because both are special paraunitary subband coders.

Using Lemma 1, we have that $\Sigma_{i=0}^{M-1} \sigma_{v_{i}}^{2}=\sum_{i=0}^{M-1} \sigma_{y_{i}}^{2}$, where $\sigma_{x_{i}}^{2}$ is the variance of $i$ th element of $\boldsymbol{x}(n)$. Hence the numerator in (2.10) is completely determined by the input statistics. The problem therefore is to minimize $\left(\Pi_{i=0}^{M-1} \sigma_{t^{\prime}}^{2}\right)^{1 / M}$ in the denominator of $(2.10)$. For a twochannel system, this is equivalent to minimizing $\sigma_{l^{\prime} 1}^{2}$. For the general case, let $\boldsymbol{R}_{x x}(i)$ and $\boldsymbol{R}_{v v}(i)$ denote the autocorrelation matrices of the vector random sequences $x(n)$ and $v(n)$, respectively. From matrix theory, we know that

$$
\operatorname{det}\left(\boldsymbol{R}_{v v}(0)\right) \leq\left(\begin{array}{ll}
M-1 & \\
\prod_{i=0}^{2} & \sigma_{i_{i}}^{2}
\end{array}\right)
$$

with equality if and only if the matrix $R_{v v}(0)$ is diagonal. In transform coding case, det $\left(\boldsymbol{R}_{v v}(0)\right)=\operatorname{det}\left(\boldsymbol{R}_{x x}(0)\right)$, and is hence determined by the input statistics. The coding gain $G_{\mathrm{TC}}$ is maximized by making det $\left(\boldsymbol{R}_{\boldsymbol{v} v}(0)\right)=$ $\left(\Pi_{i=0}^{M-1} \sigma_{t^{\prime}}^{2}\right)$, i.e., by choosing the transform to diagonalize $\left.\boldsymbol{R}_{x x}(0)\right)$. This is done by the KLT. In paraunitary subband coding, however, det $\left(\boldsymbol{R}_{v v}(0)\right)$ is not invariant, and can in fact be made less than det $\left(\boldsymbol{R}_{x x}(0)\right)$. Thus the problem is to choose the paraunitary transformation $\boldsymbol{E}(z)$ (of a fixed degree) so as to minimize $\operatorname{det}\left(\boldsymbol{R}_{\boldsymbol{w}}(0)\right)$.

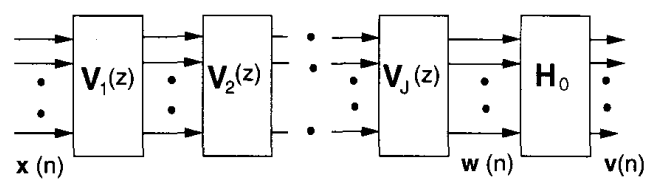

Fig. 4. Cascade implementation of a paraunitary matrix.

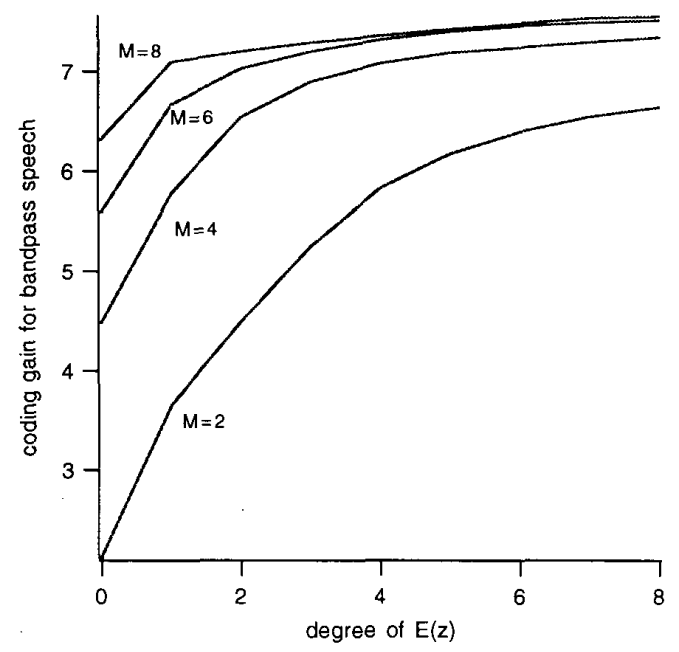

Fig. 5. Coding gain plots for bandpass speech.

From [17], we know that every FIR paraunitary matrix $\boldsymbol{E}(z)$ of degree $J$ can be written as

$$
\boldsymbol{E}(z)=\boldsymbol{H}_{0} \boldsymbol{V}_{J}(z) \boldsymbol{V}_{J-1}(z) \cdots \boldsymbol{V}_{1}(z) .
$$

Here, $\boldsymbol{H}_{0}$ is a constant unitary matrix, and the $\boldsymbol{V}_{i}(z)$ are degree-one paraunitary systems of the form

$$
\boldsymbol{V}_{i}(z)=\boldsymbol{I}-\boldsymbol{v}_{i} \boldsymbol{v}_{i}^{\dagger}+\boldsymbol{v}_{i} \boldsymbol{v}_{i}^{\dagger} z^{-1}
$$

where $\boldsymbol{v}_{i}$ are unit norm vectors (Fig. 4). Thus the unit norm vectors $\boldsymbol{v}_{i}$ and the constant orthogonal matrix $\boldsymbol{H}_{0}$ completely specify the paraunitary system.

The proposed optimization of the coding gain proceeds as follows. With reference to Fig. 4 , for given input statistics, it is possible to evaluate the det $\left(\boldsymbol{R}_{w^{\prime} \cdot}(0)\right)$ in terms of the system parameters (vectors $\boldsymbol{v}_{i}$ ). Minimization of this determinant can then be carried out using an iterative minimization scheme such as the one based on the quasiNewton techniques. We used a standard subroutine EO4JAF from the NAG Fortran library [18].

After having carried out the minimization of the said determinant, the final block in Fig. 4, which is the constant unitary matrix $\boldsymbol{H}_{0}$ is chosen to be the KLT matrix whose columns are the eigenvectors of the matrix $\boldsymbol{R}_{w^{*} \cdot}(0)$. $\boldsymbol{H}_{0}$ cannot alter the value of $\operatorname{det}\left(\boldsymbol{R}_{\boldsymbol{w} \cdot \boldsymbol{*}}(0)\right)$, and hence the choice of $\boldsymbol{H}_{0}$ does not enter into the optimization process directly.

Experimental Results: Figs. 5 and 6 show the maximum possible coding gain of paraunitary subband coders 


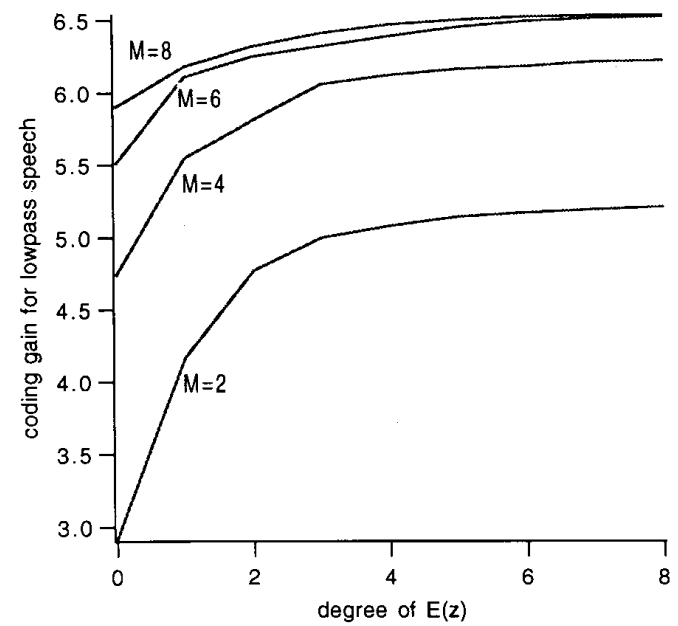

Fig. 6. Coding gain plots for low-pass speech.

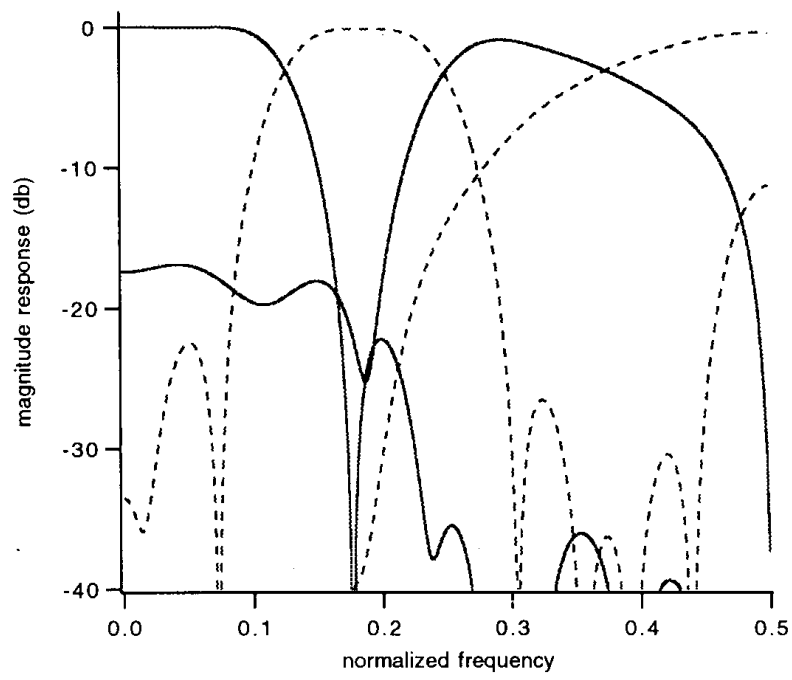

Fig. 7. Filter responses after optimizing for coding gain.

for different number of channels $M$. The abscissa indicates the degree of the paraunitary transformation $J$. Hence the length of the filters in each case is less than or equal to $M(J+1)$. A paraunitary transformation of order zero implies the usual KLT. The input in Fig. 5 was bandpass speech, whereas in Fig. 6 it was low-pass speech. Fig. 7 shows the responses of the resulting filters after optimizing for the coding gain. They correspond to the case in which the input was low-pass speech.

From the plots, we find that it is possible to achieve significant improvements in the coding gain by using paraunitary transformations. Moreover, the optimal coding gain seems to saturate quickly with increasing degree of the paraunitary transformation, so that it is sufficient to use transformations of small degrees.
III. The Discrete-Time Wavelet Transform

Now consider a further extension of the subband coding idea described above, namely, tree-structured filter banks [19], [20]. Fig. 8 shows a special case of a 3-level, binary tree-structured filter bank, drawn in terms of the polyphase matrices of the filters on each level; but the following discussion holds for a general $L$-level binary treestructured filter bank.

The input signal $x(n)$ is coded (or transformed) by passing it through the analysis bank (Fig. 8(a)). The synthesis bank (Fig. 8(b)) performs the inverse transformation on the quantized versions of $y_{k}(n)$. There are several ways to ensure that this system has the perfect-reconstruction property. One of these is to choose the analysis filters $G_{a i}(z), H_{a i}(z)$ such that their polyphase matrix $E_{i}(z)$ is paraunitary and then choose the synthesis filters as

$$
\begin{array}{r}
G_{s i}(z)=\tilde{G}_{a i}(z), \quad H_{s_{i}}(z)=\tilde{H}_{a_{i}}(z) \\
i=0, \cdots, L-1 .
\end{array}
$$

Choosing the synthesis filters in this manner means that the polyphase matrices $\boldsymbol{R}_{i}(z)$ corresponding to the synthesis filters are also paraunitary. It also means that the synthesis filters are non-causal, but since they are FIR, noncausality does not matter.

The relation between the above filter bank system and wavelet transforms has been known for quite some time [12], [21]-[24]. With reference to Fig. 8(a), the quantities $y_{k}(n)$ are called the wavelet transform coefficients of the signal $x(n)$. Assuming that perfect-reconstruction property holds, (in the absence of quantizers) we have $y(n)=x(n)$, and we can express

$$
\begin{aligned}
x(n)= & \sum_{k=0}^{L-1} \sum_{m=-\infty}^{\infty} y_{k}(m) f_{k}^{(s)}\left(n-2^{k+1} m\right) \\
& +\sum_{m=-\infty}^{\infty} y_{L}(m) f_{L}^{(s)}\left(n-2^{L} m\right)
\end{aligned}
$$

with $f_{k}^{(s)}(n)$ being the impulse response of the filter $F_{k}^{(s)}(z)$. In other words, we have obtained an expansion for the signal $x(k)$ in terms of the wavelet coefficients $y_{k}(n)$ and the wavelet basis functions $f_{k}^{(s)}\left(n-2^{k+1} m\right)$.

In the above perfect-reconstruction system, if the polyphase matrices at each level of the analysis bank are paraunitary, then the wavelet basis can be shown to be orthonormal [23], which is often a desirable property. The basis functions then satisfy the relations [23]

$$
\begin{aligned}
& \quad \sum_{n=-\infty}^{\infty} f_{k}^{(s)}\left(n-2^{k+1} m\right) f_{i}^{(s) *}\left(n-2^{l+1} i\right) \\
& \quad=\delta(k-l) \delta(m-i), \quad k, l=0, \cdots, L-1 \\
& \sum_{n=-\infty}^{\infty} f_{k}^{(s)}\left(n-2^{k+1} m\right) f_{L}^{(s) *}\left(n-2^{L} i\right)=0 \\
& \quad k=0, \cdots, L-1 \\
& \sum_{n=-\infty}^{\infty} f_{L}^{(s)}\left(n-2^{L} m\right) f_{L}^{(s) *}\left(n-2^{L} i\right)=\delta(m-i)
\end{aligned}
$$




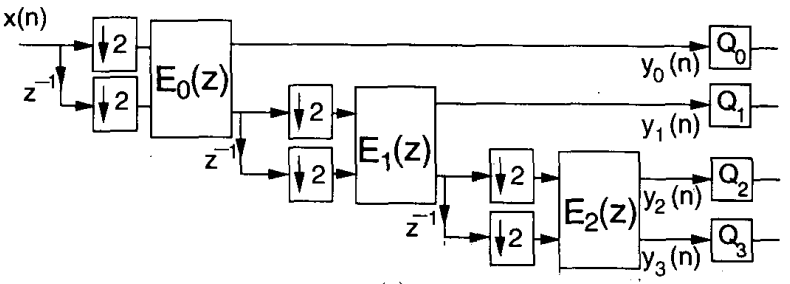

(a)

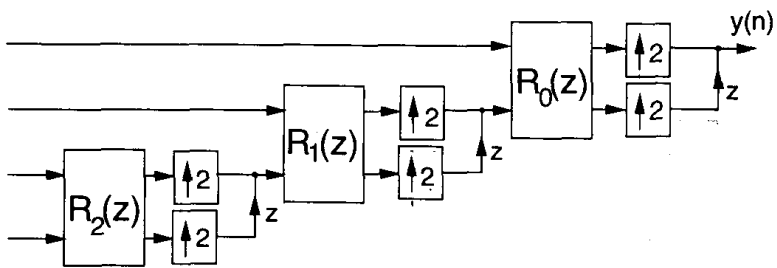

(b)

Fig. 8. A binary tree-structured filter bank in terms of the polyphase matrices. (a) The analysis bank. (b) The synthesis bank.

Here $f_{l}^{(s) *}(n)$ is obtained from $f_{l}^{(s)}(n)$ by conjugation of coefficients.

For a $L$-level binary tree, let $x(n)$ be the $2^{L}$-fold blocked version of the input $x(n)$, and $y(n)$ be the $2^{L}$-fold blocked version of the output $y(n)$. Let $\boldsymbol{r}(n)$ be the error vector as defined in (1.2). We now repeat our question on optimal bit allocation: assuming that the polyphase matrices on each level of the binary tree are paraunitary, and with proper statistical assumptions, what is the optimal way to allocate the bits among the quantizers (with their total number being fixed), so that the reconstruction error variance is minimized? We will answer this question in Theorem 2. Our result can be extended to any perfectreconstruction sub-band coding system with paraunitary polyphase matrices.

\section{A. Bit Allocation Result}

We first state two lemmas which shall be used in the proof of our main result of this section.

Lemma 2: Let $\boldsymbol{R}(z)$ be a $N$ by $N$ paraunitary matrix. Then its $M$-fold blocked version $\boldsymbol{B}(z)$ as defined in (1.17) is also paraunitary.

Proof: Consider the system shown in Fig. 9(a). $s(n)$ and $\boldsymbol{y}(n)$ are vectors of size $N$. Since the matrix $\boldsymbol{R}(z)$ is paraunitary, we have

$$
\sum_{n} y^{T}(n) y(n)=\sum_{n} s^{T}(n) s(n) .
$$

Now consider the system in Fig. $9(\mathrm{~b}), \boldsymbol{B}(z)$ is the $M$-fold blocked version of $\boldsymbol{R}(z)$. Let $\boldsymbol{p}(n)$ be the $M$-fold blocked version of $s(n)$, i.e.,

$$
p^{T}(n)=\left[s^{T}(n M) s^{T}(n M-1) \cdots s^{T}(n M-M+1)\right]
$$

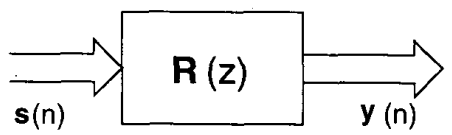

(a)

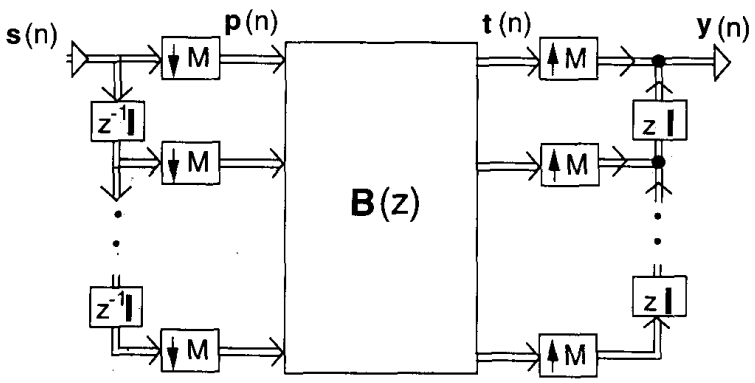

(b)

Fig. 9. (a) A MIMO system and (b) its $M$-fold blocked version.

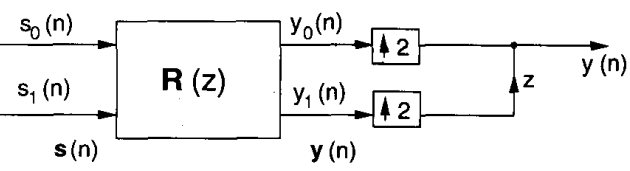

(a)

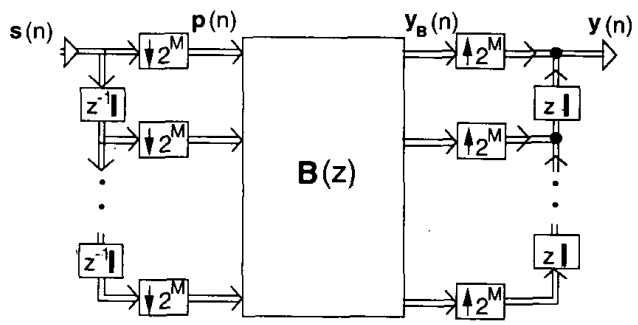

(b)

Fig. 10. (a) A two-input one output system. (b) Blocked version of the system in (a).

and let $t(n)$ be the $M$-fold blocked form of the corresponding output $\boldsymbol{y}(n)$, i.e.,

$t^{T}(n)=\left[y^{T}(n M) y^{T}(n M-1) \cdots y^{T}(n M-M+1)\right]$.

$p(n)$ and $t(n)$ are both vectors of length $n M$. For an arbitrary vector sequence of length $M$ input to the system $\boldsymbol{R}(z)$ in Fig. 9(a), the total energy at the output equals the total input energy. Thus for the system in Fig. 9(b), the output energy equals the input energy for any input $p(n)$, and so $\boldsymbol{B}(z)$ is paraunitary [25].

Lemma 3: Consider the system in Fig. 10(a):

a) Let $s^{T}(n)=\left[s_{0}(n) s_{1}(n)\right]$. Then, if $s(n)$ is a $(\mathrm{CWSS})_{2 \mathrm{M}}$ vector process, $y(n)$ is (CWSS $)_{2 \mathrm{M}+\mathrm{i}}$.

b) Further, let $s_{0}(n)$ and $s_{1}(n)$ denote vectors of length $2^{M}$ formed by blocking $s_{0}(n)$ and $s_{1}(n)$, respectively, and $y_{B}(n)$ denote the vector of length $2^{M+i}$ formed by block- 
ing $y(n)$. Then, if $\boldsymbol{R}(z)$ is paraunitary,

$$
E\left[y_{B}^{T}(n) y_{B}(n)\right]=E\left[s_{0}^{T}(n) s_{0}(n)\right]+E\left[s_{1}^{T}(n) s_{2}(n)\right] \text {. }
$$

Proof: Note that $\boldsymbol{y}_{B}(n)$ can alternatively be defined as the $2^{M}$-fold blocked version of the vector process $\boldsymbol{y}(n)$ shown in Fig. 10(a). We can redraw the system in Fig. 10(a) as in Fig. 10(b), where $p(n)$ and $y_{B}(n)$ are vectors of length $2^{M+1}$. Also, notice that $p(n)$, the $2^{M}$-fold blocked version of $s(n)$ is a WSS vector process.

The matrix $\boldsymbol{B}(z)$ is

$$
\begin{gathered}
\boldsymbol{B}(z)=\left[\begin{array}{cc}
\boldsymbol{R}_{0}(z) & \boldsymbol{R}_{1} \\
z^{-1} \boldsymbol{R}_{2 M-1}(z) & \boldsymbol{R}_{0} \\
z^{-1} \boldsymbol{R}_{2 M-2}(z) & z^{-1} \\
\vdots & \\
z^{-1} \boldsymbol{R}_{1}(z) & \cdots
\end{array}\right. \\
\text { ase components of the origi } \\
\end{gathered}
$$$$
+z^{-\left(2^{M}-1\right)} \boldsymbol{R}_{2 M-1}\left(z^{2^{M}}\right) \text {. }
$$

Since $y_{B}(n)$ is obtained by passing $\boldsymbol{p}(n)$ through a linear system, $y_{B}(n)$ is also WSS. Hence $y(n)$, which is formed by unblocking $y_{B}(n)$ is (CWSS) $)_{2 \mathrm{M}+1}$, proving part (a).

Note that since $\boldsymbol{B}(z)$ is a blocked version of a paraunitary matrix, it is also paraunitary (Lemma 2 ). Therefore using Lemma 1, we get

$$
E\left[\boldsymbol{y}_{B}^{T}(n) \boldsymbol{y}_{B}(n)\right]=E\left[\boldsymbol{p}^{T}(n) \boldsymbol{p}(n)\right] .
$$

But from the definition of these quantities,

$$
E\left[\boldsymbol{p}^{T}(n) \boldsymbol{p}(n)\right]=E\left[\boldsymbol{s}_{0}^{T}(n) s_{0}(n)\right]+E\left[\boldsymbol{s}_{1}^{T}(n) s_{1}(n)\right] .
$$

Therefore

$$
E\left[y_{B}^{T}(n) y_{B}(n)\right]=E\left[s_{0}^{T}(n) s_{0}(n)\right]+E\left[s_{1}^{T}(n) s_{1}(n)\right]
$$

proving part (b).

We now present the main result of this section. Consider a general $L$-level tree structured FIR-filter bank such as one used for generating wavelet basis functions (Fig. 8 shows a special case of a three-level binary tree). Let the polyphase matrices $\boldsymbol{E}_{i}(z)$ be paraunitary, and let $\boldsymbol{R}_{i}(z)$ $=\tilde{\boldsymbol{E}}_{i}(z)$, so that $y(n)=x(n)$ in the absence of quantizers. Let the quantizers be modeled as zero-mean, WSS, mutually uncorrelated noise sources. Let the total bit rate be constant.

Theorem 2: The reconstruction error variance of the binary tree-structured filter bank is minimized when the variances $\sigma_{i}^{2}$ of the errors introduced by each of the quantizers are equal, i.e., $\sigma_{i}^{2}=\sigma^{2}$ for all $i$.

Proof: Consider a general tree-structured analysissynthesis system. The polyphase matrices $\boldsymbol{E}_{i}(z)$ and $\boldsymbol{R}_{i}(z)$ on each level are assumed paraunitary. The bits allocated to each quantizer $R_{i}$ are related to the variance of the error introduced by that quantizer $\sigma_{i}^{2}$ as in (1.5).

Since the system in the absence of quantizers performs perfect reconstruction, and since we have assumed that noise and signal are uncorrelated, it is sufficient to con-

sider only the synthesis bank to study the effect of noise on the final reconstruction error. Consider the system in Fig. 11. The error-signals $e_{i}(n)$ are all WSS. Hence in particular, they are (CWSS $)_{M}$ for any integer $M$.

By Lemma 3, we know that $s_{L-1}(n)$ is (CWSS) $)_{2}$. Now, $e_{L-2}(n)$ is also (CWSS) $)_{2}$ and $e_{L-2}(n)$ and $s_{L-2}(n)$ are uncorrelated, and hence they are jointly (CWSS $)_{2}$. Applying Lemma 3 again, we have that $s_{L-2}(n)$ is (CWSS $)_{4}$. In general, $e_{k}(n)$ and $s_{k+1}(n)$ are jointly $(\mathrm{CWSS})_{2}{ }^{L-k-1}$, hence $s_{k}(n)$ is $(\text { CWSS })_{2} L-k$.

Let $\boldsymbol{e}_{k}(n)$ and $\boldsymbol{s}_{k+1}(n)$ denote the $2^{L-k-1}$-fold blocked versions of $e_{k}(n)$ and $s_{k+1}(n)$, respectively, for $k=0$, $\cdots, L-2$; for example, $s_{0}(n)$ is a $2^{L}$-fold blocked version of $s_{0}(n)$, whereas $e_{0}(n)$ and $s_{1}(n)$ are $2^{L-1}$ fold blocked versions of $e_{0}(n)$ and $s_{1}(n)$, respectively, and so on. The tree can be redrawn in terms of the polyphase matrices on all levels, similar to Fig. 8.

By applying Lemma 3 to each level of the tree, we get the following set of equalities:

$$
\begin{aligned}
& E\left[\boldsymbol{y}^{T}(n) \boldsymbol{y}(n)\right]=\left(E\left[\boldsymbol{e}_{0}^{T}(n) \boldsymbol{e}_{0}(n)\right]+E\left[\boldsymbol{s}_{1}^{T}(n) \boldsymbol{s}_{1}(n)\right]\right) \\
& E\left[\boldsymbol{s}_{1}^{T}(n) \boldsymbol{s}_{1}(n)\right]=\left(E\left[\boldsymbol{e}_{1}^{T}(n) \boldsymbol{e}_{1}(n)\right]+E\left[\boldsymbol{s}_{2}^{T}(n) \boldsymbol{s}_{2}(n)\right]\right) \\
& E\left[\boldsymbol{s}_{2}^{T}(n) \boldsymbol{s}_{2}(n)\right]=\left(E\left[\boldsymbol{e}_{2}^{T}(n) \boldsymbol{e}_{2}(n)\right]+E\left[\boldsymbol{s}_{3}^{T}(n) \boldsymbol{s}_{3}(n)\right]\right), \\
& \quad \text { in general, } \\
& E\left[\boldsymbol{s}_{k}^{T}(n) \boldsymbol{s}_{k}(n)\right]=\left(E\left[\boldsymbol{e}_{k}^{T}(n) \boldsymbol{e}_{k}(n)\right]+E\left[\boldsymbol{s}_{k+1}^{T}(n) \boldsymbol{s}_{k+1}(n)\right]\right), \\
& k=0, \cdots, L-2
\end{aligned}
$$

and finally,

$$
E\left[s_{L-1}^{T}(n) s_{L-1}(n)\right]=\left(E\left[e_{L-1}^{2}(n)\right]+E\left[e^{2} L(n)\right]\right) .
$$




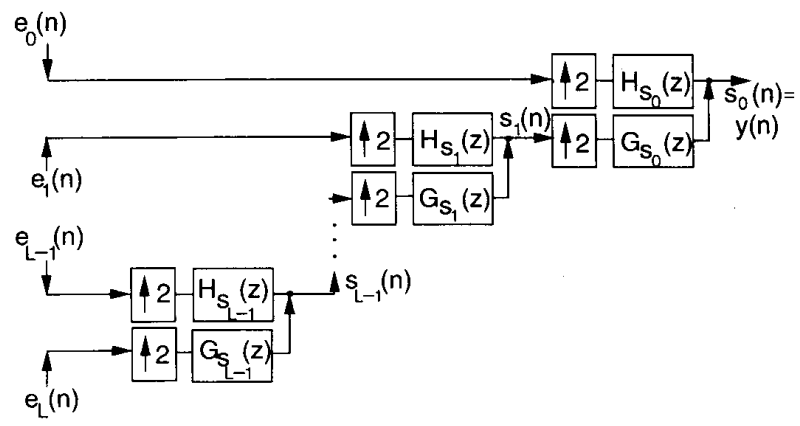

Fig. 11. A binary tree-structured synthesis bank.

Hence from the above equations, one can write,

$$
\begin{aligned}
E\left[y^{T}(n) y(n)\right]= & E\left[e_{0}^{T}(n) e_{0}(n)\right]+E\left[e_{1}^{T}(n) e_{1}(n)\right] \\
& +E\left[e_{2}^{T}(n) e_{2}(n)\right] \\
& +\cdots+E\left[e_{L-1}(n) e_{L-1}(n)\right] \\
& \left.+E\left[e_{L}(n) e_{L}(n)\right]\right)
\end{aligned}
$$

i.e., the variance of the overall reconstruction error $\left(1 / 2^{L}\right) E\left[y^{T}(n) y(n)\right]=\sigma_{r}^{2}$ is given as

$$
\begin{aligned}
\sigma_{r}^{2}= & \left(\frac{1}{2^{L}}\right)\left(2^{L-1} \sigma_{0}^{2}+2^{L-2} \sigma_{1}^{2}+2^{L-3} \sigma_{2}^{2}+\cdots\right. \\
& \left.+\sigma_{L-1}^{2}+\sigma_{L}^{2}\right)
\end{aligned}
$$

where $\sigma_{i}^{2}$ are the individual quantizer variances. Our constraint is that the total bit rate is constant. This means that

$$
\begin{aligned}
& \left(2^{L-1} R_{0}+2^{L-2} R_{1}+2^{L-3} R_{2}+\cdots+R_{L-1}+R_{L}\right) / 2^{L} \\
& \quad=\text { constant }=R .
\end{aligned}
$$

To obtain the optimal bit allocation, we minimize the reconstruction error variance $\sigma_{r}^{2}$ in $(3.15)$ under the above constraint on the bit rate. This can be done by the method of Lagrange multipliers, and it gives the following optimal bit allocation:

$$
R_{i}=R+(1 / 2) \log _{2}\left[\frac{\sigma_{v_{i}}^{2}}{D}\right] \quad i=0, \cdots, L
$$

where

$$
D=\left(\sigma_{l^{\prime} L}^{2}\right)^{1 / 2^{L}} \prod_{j=0}^{L-1}\left(\sigma_{l^{\prime}}^{2}\right)^{1 / 2^{j+1}}
$$

and $\sigma_{v_{i}}^{2}$ are the variances of the inputs to the quantizers. It can be verified that under optimal bit allocation the variances of the errors introduced by each of the quantizers are equal.

As in the case of Theorem 1, the above result is valid only for high bit rates.

The total reconstruction error variance under optimal bit allocation becomes

$$
\sigma_{r}^{2}=\epsilon^{2} D 2^{-2 R}
$$

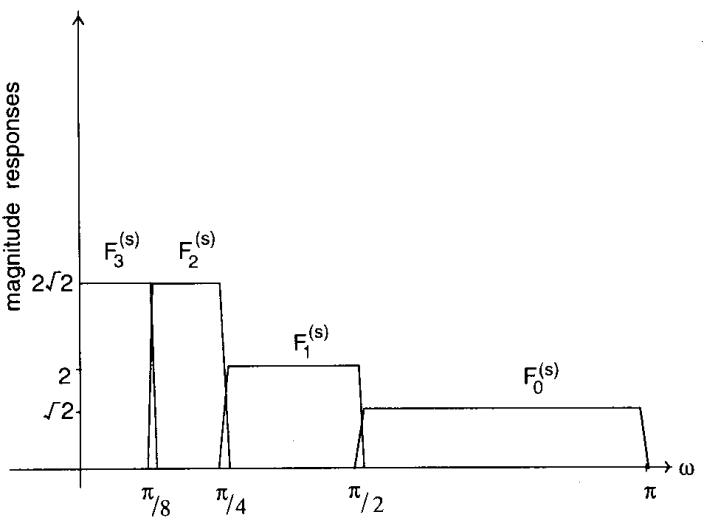

Fig. 12. Typical appearances of the magnitude responses of synthesis filters in a 3 -level tree.

The coding gain of the system is therefore,

$$
G_{P U}=\sigma_{x}^{2} / D
$$

where $\sigma_{r}^{2}$ is the variance of the input.

To obtain a physical insight into this result, consider the typical appearances of the filter responses in a treestructured bank (Fig. 12, for a 3-level tree). If the polyphase matrices on each level of the tree are paraunitary, these filters have equal energy irrespective of their frequency characteristics. This can be proved as follows. Putting $k=l$ and $m=i=0$ in (3.3) we get

$$
\sum_{n=-\infty}^{\infty} f_{k}^{(s)}(n) f_{k}^{(s) *}(n)=1, \quad k=0, \cdots, L
$$

This means that all filters have unit energy. Hence it is indeed appealing intuitively to equalize the variance of the error in each subband.

Notice that in the proof of the above result, the assumption that the noise sources are mutually uncorrelated is a slightly stronger assumption than we actually need. It would have been enough to assume that the vectors $s_{k}(n)$, $k=0, \cdots, L-1$ are WSS.

\section{Bit Allocation in Orthonormal Nonuniform FILTER BANKS}

Consider the filter bank shown in Fig. 13. This is an example of a general nonuniform filter bank. The numbers $n_{i}$ need not necessarily be powers of 2 , as they were in the case of the discrete-time wavelet transforms. Nonuniform filter banks with perfect reconstruction property have been shown to exist. The filter bank is said to be orthogonal, if the synthesis filters satisfy the following condition [26]

$$
\sum_{n=-\infty}^{\infty} f_{k}^{(s)}(n) f_{l}^{(s) *}(n-g i)=\delta(k-l) \delta(i)
$$

where $g$ is the greatest common divisor of $\left(n_{k}, n_{l}\right)$. One way to realize perfect reconstruction nonuniform filter banks is to generate them via tree structures. Orthonor- 


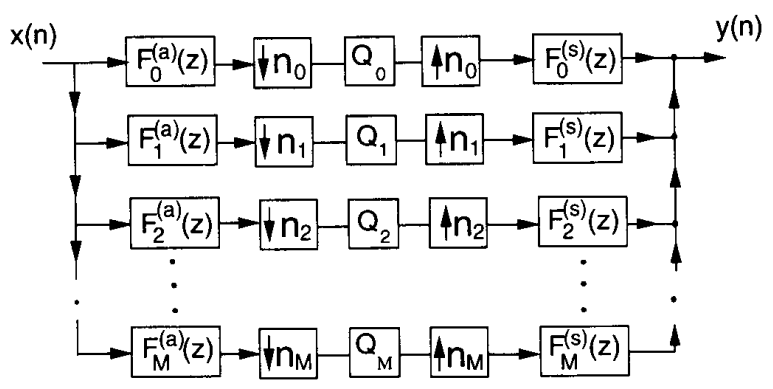

Fig. 13. A nonuniform filter bank.

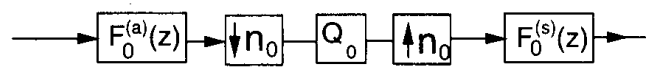

(a)

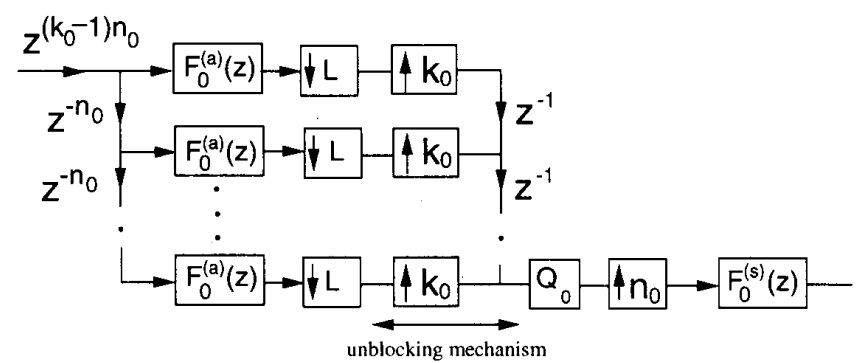

(b)

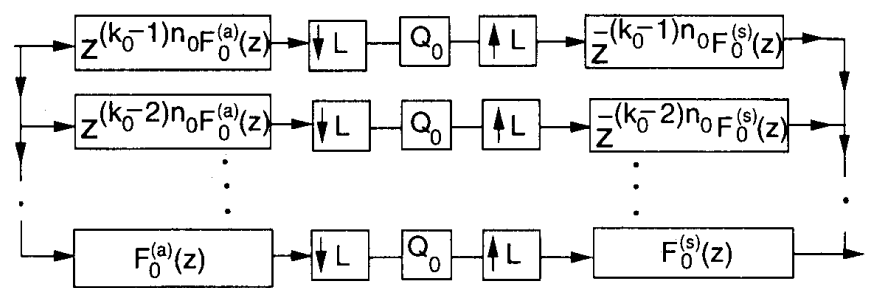

(c)

Fig. 14. Transforming a nonuniform filter bank to a uniform one.

mality of the resulting nonuniform system can be ensured by choosing the polyphase matrices on each level to be paraunitary [26]. Hence, the analysis of the previous section can be extended in a straightforward manner to orthogonal nonuniform filter banks which arise from tree structures. However, it must be noted that not all orthonormal nonuniform filter banks can be generated using tree structures [26]. In this case, a different approach needs to be taken to arrive at the bit-allocation results. This is the topic of the present section.

The trick is to reduce the orthogonal nonuniform filter bank shown in Fig. 13 to a uniform paraunitary filter bank. This idea has found mention in [27]. This will then enable us to directly use the results developed in Section II.

Let $L$ be the least common multiplier of the decimation ratios $n_{i}$, and let $L=n_{i} k_{i}, i=0, \cdots, M$. It can be verified that $L=\sum_{i=0}^{M} k_{i}$. Now consider one branch of the nonuniform bank, as shown in Fig. 14(a). This can be redrawn as in Fig. 14(b). The unblocking mechanism shown only interleaves the samples (i.e., no addition of two nonzero samples takes place). Hence the quantizer can be moved across the unblocking mechanism into each of the branches. Finally, the individual branch we started with can be redrawn as in Fig. 14(c). If we use the preceding technique to represent all branches of the nonuniform filter bank, the resulting system is a $L$-channel uniform filter bank. It can be shown [28] that if the original nonuniform filter bank is orthogonal, the polyphase matrix corresponding to the new $L$-channel uniform filter bank is paraunitary. Hence, applying Lemma 1 to the uniform system, we get

$$
\sigma_{r}^{2}=(1 / L) \sum_{i=0}^{M} k_{i} \sigma_{i}^{2}
$$


where $\sigma_{i}^{2}$ is the variance of the error introduced by the $i$ th quantizer in Fig. 13.

The problem is to minimize this reconstruction error variance under the constraint of constant bit rate, namely,

$$
\left(\sum_{i=0}^{M} k_{i} R_{i}\right) / L=\text { constant }=R .
$$

The optimal bit allocation is again found by the Lagrange multiplier method. For optimality, the number of bits allocated to the $i$ th quantizer is given by

$$
R_{i}=R+(1 / 2) \log _{2}\left[\frac{\sigma_{v^{\prime} i}^{2}}{D_{n}}\right] \quad i=0, \cdots, M
$$

where

$$
D_{n}=\prod_{j=0}^{M}\left(\sigma_{v_{j}}^{2}\right)^{1 / n_{j}} .
$$

The $\sigma_{v_{i}}^{2}$ are usual, the variances of the inputs to the quantizers. Once again, it can be verified that under this condition the variance of the errors at the location of each of the quantizers are equal.

The total reconstruction error variance under optimal bit allocation is given by

$$
\sigma_{r}^{2}=\epsilon^{2} D_{n} 2^{-2 R} \text {. }
$$

The coding gain of the system is, therefore,

$$
G_{\mathrm{PU}}=\sigma_{x}^{2} / D_{n}
$$

where $\sigma_{x}^{2}$ is the variance of the input.

\section{Conclusions}

In this paper we have proved results for bit allocation in subband coding schemes using paraunitary matrices. To start with, we proved some basic results for simple paraunitary LTI systems. Using these we have then derived bit-allocation results for a more complex system, namely, the tree-structured filter bank. For a binary treestructured filter bank, we showed that under the constraint that the total bit rate is fixed, the individual quantizer error variances have to be equal under optimal bit allocation. These theorems can be extended readily to general tree-structures. We finally performed this analysis for nonuniform orthogonal filter banks which cannot be derived from tree structures. It would also be possible to parametrize wavelet filter banks in terms of the polyphase matrices on each level of the tree structure and optimize the overall coding gain, though this has not been done in this paper. This procedure would result in an optimized wavelet for the given signal statistics.

In the case of uniform paraunitary subband coders, we presented a scheme to directly optimize the coding gain. Experimental results were presented.

In speech and image coding applications, one might use other criterion for minimizing the reconstruction error, rather than minimizing its variance. For instance, we could attach different (nonnegative) weights $w_{k}$ to each of the subbands, and then try to minimize the weighted sum of the variances, i.e., $\Sigma_{k} w_{k} \sigma_{k}^{2}$. The fact that the arithmetic mean of a set of nonnegative numbers is always greater than or equal to their geometric mean can still be used. It is easy to see that in order to minimize the weighted sum, each of the $w_{k} \sigma_{k}^{2}$ would have to be equal. In other words, the variances $\sigma_{k}^{2}$ would have to be inversely proportional to the weights $w_{k}$, again an intuitively appealing result.

\section{ACKNOWLEDGMENT}

The authors are grateful to Dr. V. Sathe, General Instrument, for several useful comments on the topic of this paper. They also wish to acknowledge the comments of the anonymous reviewers.

\section{REFERENCES}

[1] J. J. Huang and P. M. Schultheiss, "Block quantization of correlated Gaussian random variables," IEEE Trans. Commun. Syst.. pp. 289296, Sept. 1963.

[2] N. S. Jayant and P. Noll, Digital Coding of Waveforms-Principles and Applications to Speech and Video. Englewood Cliffs, NJ: Prentice-Hall Signal Processing Series, 1984

[3] J. W. Woods and S. D. O'Neil, "Subband coding images," IEEE Trans. Acoust., Speech, Signal Processing, vol. 34, pp. 12781288, Oct. 1986.

[4] Crochiere, Multirate Digital Signal Processing. Englewood Cliffs, NJ: Prentice-Hall Signal Processing Series, 1983.

[5] C. Galand and D. Esteban, "16 Kbps real-time QMF subband coding implementation," in Proc. Int. Conf. ASSP, Denver, CO, Apr. 1980, pp. $332-335$

[6] P. P. Vaidyanathan, "Multirate digital fijters, filter banks, polyphase networks, and applications: A tutorial," Proc. IEEE, vol. 78 , no. 1 , Jan. 1990.

[7] P. P. Vaidyanathan, "Theory and design of $M$-channel maximally decimated filters with arbitrary $M$, having perfect reconstruction property." IEEE Trans. Acoust., Speech, Signal Processing, pp. 476492, Apr. 1987

[8] H. S. Malvar, "Lapped transforms for efficient transform/subband coding." IEEE Trans. Acoust., Speech, Signal Processing, vol. 38 , no. 6. pp. 969-978, June 1990

[9] A. Akansu and Liu, "On signal decomposition techniques," Opt. Eng. J., pp. 912-920, vol. 30, no. 7, July 1991

[10] W. A. Pearlman, "Performance bounds for subband coding. " in Subband Image Coding. J. Woods. Ed. Kluwer. 1991, ch. 1.

[11] A. Segall, "Bit allocation and encoding for vector sources," IEEE Trans. Inform. Theory, pp. 162-169. Mar. 1976.

[12] I. Daubechies, "Orthonormal bases of compactly supported wavelets," Commun. Pure Appl. Math., vol. 4, pp. 909-996, Nov. 1988.

[13] O. Rioul and M. Vetterli, "Wavelets and signal processing," IEEE Signal Processing Mag., pp. 14-38, Oct. 1991.

[14] P. P. Vaidyanathan and S. K. Mitra, "Polyphase networks, block digital filtering. and alias-free QMF banks: A unified approach based on pseudocirculants." IEEE Trans. Acoust., Speech, Signal Processing. vol. 36, pp. 381-391, Mar. 1988.

[15] E. F. Beckenbach and R. E. Bellman, Inequalities. Berlin: Springer, 1961 .

[16] H. S. Malvar, "Extended lapped transforms: Properties, applications, and fast algorithms," preprint.

[17] P. P. Vaidyanathan. T. Q. Nguyen, Z. Doganata, and T. Saramaki, "Improved technique for design of perfect reconstruction FIR QMF banks with lossless polyphase matrices," IEEE Trans. Acoust. Speech, Signal Processing, pp. 1042-1056, July 1989.

[18] NAG Fortran Library, Numerical Algorithms Group Ine., Downers Grove, IL.

[19] M. J. T. Smith and T. P. Barnwell, III, "A procedure for designing exact reconstruction filter banks for tree-structured subband coders," in Proc. IEEE Int. Conf. ASSP, San Diego, CA, Mar. 1984.

[20] F. Mintzer. "Filters for distortion-free two-band multirate filte 
banks," IEEE Trans. Acoust., Speech, Signal Processing, pp. 626630, June 1985

[21] M. Vetterli and C. Herley, "Wavelets and filter banks: Relationships and new resuits," in Proc. ICASSP, 1990, pp. 1723-1726.

[22] S. G. Mallat. "A theory for multiresolution signal decomposition: The wavelet representation," IEEE Trans. Patt. Anal. Machine Intell., pp. 674-693, July 1989.

[23] P. P. Vaidyanathan, "Lossless systems in wavelet transforms." in Proc. IEEE Int. Symp. Circuits Syst. Singapore, June 1991, pp. 116 119.

[24] G. Evangelista, "Orthogonal wavelet transforms and filter banks," in Proc. 23rd Asilomar Conf. 1989.

[25] P. P. Vaidyanathan and Z. Doğanata, "The role of lossless systems in modern digital signal processing: A tutorial," Special Issue on Circuits and Systems, IEEE Trans. Education, Aug. 1989.

[26] A. Soman and P. P. Vaidyanathan, "Paraunitary filter banks and wavelet packets," in Proc. ICASSP, San Francisco, 1992, pp. 397400 .

[27] P. Hoang and P. P. Vaidyanathan, "Nonuniform multirate filter banks: Theory and design," in Proc. ISCAS, Portland, 1989.

[28] I. Djokovic and P. P. Vaidyanathan, "Some results on orthonormal filter banks," preprint.

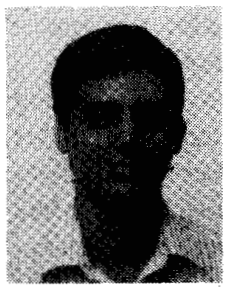

Anand K. Soman ( $S^{*} 91$ ) was born on April 20 1968 in Bombay, India. He received the B.Tech. degree in electrical engineering from the Indian Institute of Technology, Bombay, in 1989. He is currently pursuing the doctoral degree at the $\mathrm{Cal}$ ifornia Institute of Technology, Pasadena. His research interests include multirate signal processing, adaptive filtering. and digital filter design.

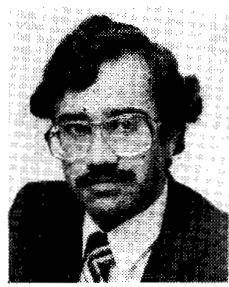

P. P. Vaidyanathan (S'80-M'83-SM'88-F'91) was born in Calcutta, India, on October 16, 1954. $\mathrm{He}$ received the B.Sc. (Hons.) degree in physics and the B.Tech. and M.Tech. degrees in radiophysics and electronics, all from the University of Calcutta, India, in 1974, 1977, and 1979, respectively, and the Ph.D. degree in electrical and computer engineering from the University of Calfornia. Santa Barbara, in 1982

He was a postdoctoral fellow at the University of California. Santa Barbara. from Septembe 1982 to March 1983. In March 1983 he joined the Department of Electrical Engineering of the California Institute of Technology as an Assistant Professor, and since 1988 has been an Associate Professor of Electrical Engineering there. His main research interests are in digital signal processing. multirate systems, wavelet transforms, and adaptive filtering.

Dr. Vaidyanathan served as Vice Chairman of the Technical Program Committee for the 1983 IEEE International Symposium on Circuits and Systems, and as an Associate Editor for the IEEE TRANSACTIONS ON CIR CUITS AND SYSTEMS for the period 1985-1987. He also served as the Tech. nical Program Chairman for the 1992 IEEE International Symposium on Circuits and Systems. He was a recipient of the Award for Excellence in Teaching at the Califormia Institute of Technology for the year 1983-1984. He also received the NSF's Presidential Young Investigator Award in 1986. In 1989 he received the IEEE ASSP Senior Award for his paper on multirate perfect-reconstruction filter banks. In 1990 he received the S. K. Mitra Memorial Award from the Institute of Electronics and Telecommunications Engineers, India, for his joint paper in the IETE journal. He was also the coauthor of a paper on linear-phase perfect reconstruction filter banks in the IEEE Transactions on Signal. Processing, for which the first author (Truong Nguyen) received the Young Outstanding Author Award in 1993. He was elected a Fellow of the IEEE in 1991. 